\title{
Segmentation of 3D Medical Structures Using Robust Ray Propagation
}

\author{
Hüseyin Tek, Martin Bergtholdt, Dorin Comaniciu, and James Williams \\ Imaging and Visualization, Siemens Corporate Research, Inc. \\ 755 College Road East, Princeton NJ 08540, USA
}

\begin{abstract}
A robust and efficient method for the segmentation of 3D structures in CT and MR images is presented. The proposed method is based on 3D ray propagation by mean shift analysis with a smoothness constraint. Specifically, ray propagation is used to guide an evolving surface due to its computational efficiency. In addition, non-parametric analysis and shape priors are incorporated to the proposed technique for robust convergence. Several examples are depicted to illustrate its effectiveness.
\end{abstract}

\section{Introduction}

The segmentation of 3D structures in CT and MR images is an inherently difficult and time consuming problem. The accurate localization of object surfaces is influenced by the presence of significant noise levels, generated through partialvolume effects and by image acquisition devices. In addition, the processing in 3D space implies an increased computational complexity, which makes impractical the 3D extension of most two-dimensional segmentation algorithms.

Deformable models have been popular for the segmentation of 3D medical images 18,5,10,17,23 22,13,24,15. These approaches can produce good segmentation results if the initialization is done properly. However, choosing proper initialization of deformable models is typically a difficult task. In addition, the current deformable model approaches are computationally expensive. This is due to the additional dimension in the case of level set methods 1249, or expensive reinitialization of parameters in the case of explicit surface evolution techniques 18,5 .

In this paper, we present an efficient, robust, and user-friendly method for the interactive segmentation of 3D medical structures, e.g., aneurysm from contrast enhanced CT or MR data. The primary innovation of the method is the boundary propagation by mean shift analysis combined with a smoothness constraint. As a result, our technique is robust to both outliers in the data and missing data structures. The first property is guaranteed by the mean shift procedure, while the second is obtained through the use of a priori information on boundary smoothness. Furthermore, due to our computationally efficient framework based on ray propagation, the entire processing is fast, allowing real-time user interaction (under 10 seconds on a Pentium III 1Ghz). A two-dimensional version of this approach was first presented in [16] for detecting 2D vessel boundaries in cross-sectional views in contrast enhanced $\mathrm{CT}$ and MR images. In this paper, we extend the technique to the segmentation of 3D medical structures and demonstrate it on aneurysms in 3D CTA data. 
Within the proposed segmentation method, the user specifies the structure to be segmented by placing a single seed inside it. A boundary surface is then automatically generated via the propagation of rays from the seed point. The propagation is guided by the image forces defined through mean shift analysis and smoothness constraints. The gradient-ascent mean shift localizes edges accurately in the presence of noise and provides good computational performance, being based on local operators. Currently, we employ the mean curvature for the smoothness constraint, however, other geometric smoothing techniques, such as the Mean-Gaussian curvature [11] can be applied. Figure 1] summarizes the goal of this paper and the method described here. Currently, we are working on validating our technique with phantoms of known ground-truth and data from clinical practice.

\section{Deformable Surfaces for 3D Volume Segmentation}

Deformable model-based segmentation algorithms can be divided into explicit methods and implicit methods based on how the deformable surface is represented. In explicit methods, deformable surfaces are represented by triangular mashes [23, superquadrics [18, and others 5108. In implicit methods, the popular choice for deformable surface representation is the level set methods. Level set methods $12,4,9,1,17,15$ have been popular because they are topologically flexible and can represent complex shapes without any computationally expensive reparametrization step. One of the main shortcomings of level set methods is the computational complexity due to the additional embedded surface requirement for simulations. To overcome the complexity, narrow band level set evolutions have been proposed [9 1 121] 1. However, these methods are still not fast enough for real-time volume segmentation. Thus, in this paper, we rely on ray propagation for fast image segmentation.

\subsection{Ray Propagation}

Let the front be represented by a $3 \mathrm{D}$ surface $\psi(\xi, \eta, t)=(x(\xi, \eta, t), y(\xi, \eta, t)$, $z(\xi, \eta, t))$ where $x, y$ and $z$ are the Cartesian coordinates, $\xi, \eta$ parameterize the surface, and $t$ is time. The front evolution is governed by

$$
\left\{\begin{array}{l}
\frac{\partial \psi}{\partial t}=S(x, y, z) \mathbf{N} \\
\psi(\xi, \eta, 0)=\psi_{0}(\xi, \eta)
\end{array}\right.
$$

where $\psi_{0}(\xi, \eta)=(x(\xi, \eta, 0), y(\xi, \eta, 0), z(\xi, \eta, 0))$ is the initial surface, and $\boldsymbol{N}$ is the unit normal vector and $S(x, y, z)$ is the speed of the surface at point $(x, y, z)$.

The approach that we consider is based on explicit front propagation via normal vectors. The surface is sampled and the evolution of each sample is followed in time by rewriting the Eikonal equation [14] in vector form, namely,

\footnotetext{
${ }^{1}$ Similarly, Sethian [14] proposed fast marching methods for simulating the monotonically advancing front. This method does not require any additional surface, but it is limited to simulate one directional flows, i.e., no curvature smoothing, thus its use in segmentation is rather limited.
} 
(a)

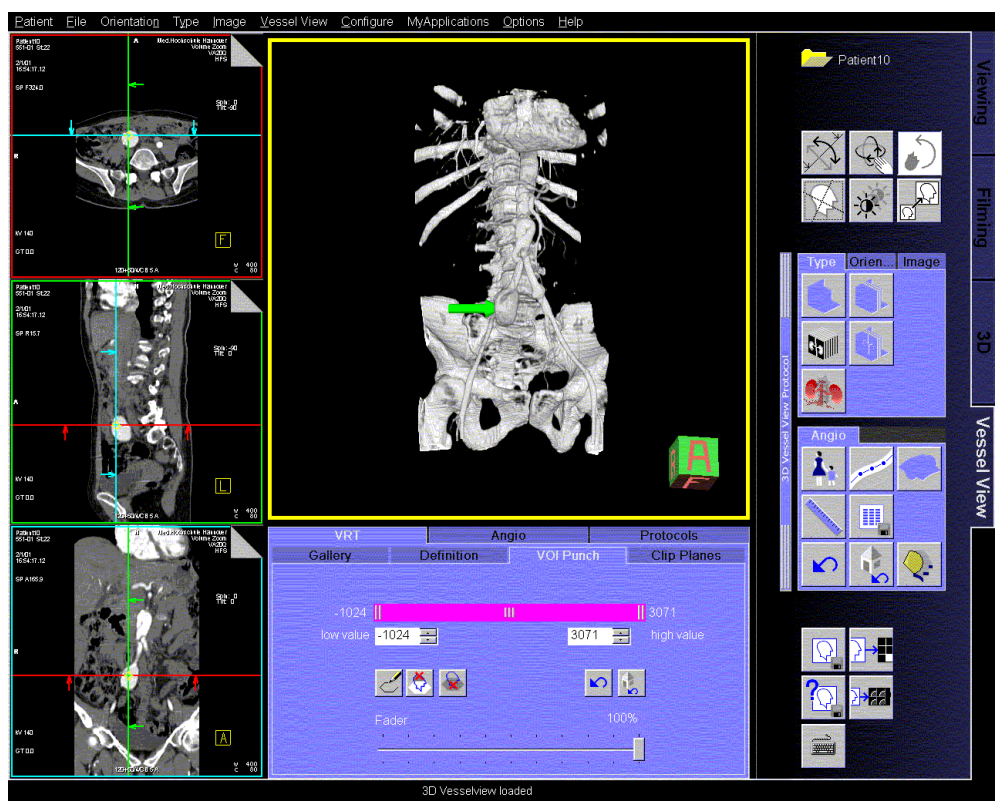

(b)
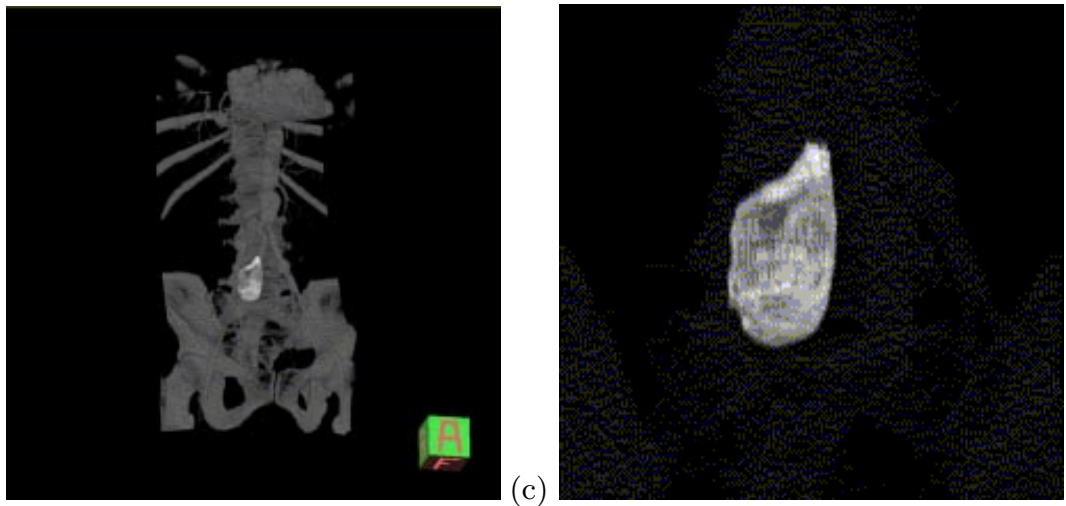

Fig. 1. This figure summarizes the method described in this paper. (a) illustrates the original (CE-CTA) data in multi-planar reformats (MPRs) and volume rendering. This data set contains an aneurysm which is indicated by the arrow. In addition, MPRs (orthogonal views) are centered on this pathology. The system allows the radiologist quickly detect pathologies from different visualizations. The next step is the quantification of these pathologies. The goal of this work is to provide a mechanism such that an user can quickly measure the volume of blob-like structures by simply clicking on them. In this example, the user clicks on a structure of interest and the $3 \mathrm{D}$ ray propagation algorithm detects the boundary of the structure and computes its volume. (b) The detected aneurysm (lumen boundary) is blended with the original data. (c) Segmented aneurysm (lumen boundary). 


$$
\left\{\begin{array}{l}
x_{t}=S(x, y, z) \frac{\boldsymbol{N}_{x}}{\|\mathbf{N}\|} \\
y_{t}=S(x, y, z) \frac{\boldsymbol{N}_{\boldsymbol{y}}}{\|\mathbf{N}\|} \\
z_{t}=S(x, y, z) \frac{\boldsymbol{N}_{z}}{\|\boldsymbol{N}\|}
\end{array}\right.
$$

This evolution is the "Lagrangian" solution since the physical coordinate system moves with the propagating wavefront. In general, the applications of ray propagation for surface evolution have been limited. Since the normals to the wavefront may collide (formation of shocks), this approach exhibits numerical instabilities due to an accumulated density of sample points, thus requiring special care, such as reparametrization of the wavefront. Also, topological changes are not handled naturally, an external procedure being required.

Previously, we have successfully used the $2 \mathrm{D}$ ray propagation method for segmentation of vessel boundary cross-sections in contrast enhanced CT and MR images [16. In this paper, we advocate ray propagation from a single point source for the segmentation of blob-like structures found in medical images, such as aneurysm and brain tumors. In fact, ray propagation is well suited for these problems because it is very fast, no topological changes are necessary and no shocks form during the propagation, since rays from a single source point do not collide with each other.

Observe that the speed function $S(x, y, z)$ plays an important role in the quality of the segmentation results. In this paper, the speed of rays, $S(x, y, z)$ depends on image information and shape priors. Specifically, we propose to use $S(x, y, z)=S_{0}(x, y, z)+\beta S_{1}(x, y, z)$ where $S_{0}(x, y, z)$ measures image discontinuities, $S_{1}(x, y, z)$ represents shape priors, and $\beta$ balances these two terms.

\subsection{Robust Measure of Discontinuities: Mean-Shift Analysis}

Traditionally, image gradients are used to guide deformable models to object boundaries 49. However, they are not robust to noise, and outliers in the data can significantly influence the result. A solution to this problem is to use robust measures of discontinuities.

Our approach is based on a new representation of image data which uses kernel density estimation 677. Let us consider a 1-dimensional intensity profile (ray) obtained from a gray level image. Each pixel along the ray is characterized by a location $x$ and an intensity value $I$. As a result, an input ray of $N$ pixels is represented as a collection of 2 -dimensional points $\left\{x_{i}, I_{i}\right\}_{i=1, \ldots, N}$. The 2-dimensional space constructed as before is called the joint spatial-intensity domain. Note that this construction generalizes for standard gray-level image data (3-dimensional space with 2 dimensions for coordinates and one for intensity), color data (5-dimensional space with 2 dimensions for coordinates and three for color), multi-modal data, or image sequences.

The task of identifying image discontinuities reduces to the partitioning of the joint space according to the probability density estimated in this space. The number of segments is determined by the number of peaks (modes) of the underlying density. The iterative mean shift procedure [7] is used to find the modes in the joint space and assign each data point to a mode in its neighborhood. Denote by $\left\{x^{*}{ }_{i}, I^{*}{ }_{i}\right\}$ the mode associated to $\left\{x_{i}, I_{i}\right\}$ where $i=1, \ldots, N$. Since the modes delineate regions with high concentration of similar data, the robustness 
to outliers is guaranteed. The overall analysis is related to robust anisotropic diffusion [3] and bilateral filtering [19].

Let $\left\{d_{i}=x^{*}{ }_{i}-x_{i}\right\}_{i=1, \ldots, N}$ be the oriented spatial distance from a point to its assigned mode. We will further refer to this quantity as the displacement vector. The displacement vectors always point away from discontinuities and they play a dominant role in our propagation technique. The iterative, mean shift-based algorithm for finding the displacement vectors is

For each $i=1, \ldots, N$

1. Initialize $k=1$ and $\left(x_{i}^{k}, I_{i}^{k}, d_{i}\right)=\left(x_{i}, I_{i}, 0\right)$

2. Compute

$$
\begin{aligned}
x_{i}^{k+1}= & \frac{\sum_{j=1}^{M} x_{j} e^{\frac{-\left(x_{i}^{k}-x_{j}\right)^{2}}{2 \sigma_{x}^{2}}} e^{\frac{-\left(I_{i}^{k}-I_{j}\right)^{2}}{2 \sigma_{I}^{2}}}}{\sum_{j=1}^{M} e^{\frac{-\left(x_{i}^{k}-x_{j}\right)^{2}}{2 \sigma_{x}^{2}}} e^{\frac{-\left(I_{i}^{k}-I_{j}\right)^{2}}{2 \sigma_{I}^{2}}}} \\
I_{i}^{k+1}= & \frac{\sum_{j=1}^{M} I_{j} e^{\frac{-\left(x_{i}^{k}-x_{j}\right)^{2}}{2 \sigma_{x}^{2}}} e^{\frac{-\left(I_{i}^{k}-I_{j}\right)^{2}}{2 \sigma_{I}^{2}}}}{\sum_{j=1}^{M} e^{\frac{-\left(x_{i}^{k}-x_{j}\right)^{2}}{2 \sigma_{x}^{2}}} e^{\frac{-\left(I_{i}^{k}-I_{j}\right)^{2}}{2 \sigma_{I}^{2}}}}
\end{aligned}
$$

until convergence.

3. Assign $\left(x^{*}{ }_{i}, I^{*}{ }_{i}\right)=\left(x_{i}^{k+1}, I_{i}^{k+1}\right)$

4. Assign $d_{i}=\left(x^{*}{ }_{i}-x_{i}\right)$

where $\sigma_{x}$ and $\sigma_{I}$ are the bandwidths of Gaussian kernels in the spatial and intensity domain, respectively. For details and explanatory figures on displacement vector computation we refer the reader to [16].

The first part of the speed term can be now defined by

$$
S_{0}(x, y, z)=\frac{f(x, y, z)}{1.0+|\nabla d(x, y, z)|^{2}}
$$

The function $d(x, y, z)$ is obtained by summarizing the information provided by the displacement vectors. For a given location along a ray, $d(x, y, z)$ is positive when the corresponding displacement vector is pointing outbound and negative when the vector points towards the seed point. The term $f(x, y, z)$ is given by

$$
f(x, y, z)= \begin{cases}-\operatorname{sign}(d(x, y, z)) & \text { if }|\nabla d(x, y, z)|<\text { threshold } \\ 1 & \text { else }\end{cases}
$$

where the gradient of $d(x, y, z)$, namely $\nabla d(x, y, z)$ is computed along the ray. In this formulation, rays propagate freely towards the object boundaries when they are away from them and slow down in the vicinity of boundaries. If a ray crosses over a boundary, it returns to the boundary. The parameter threshold is determined from the statistical measures computed from the density function of displacement vectors. It is assumed that locations of small gradients (less than threshold) in the displacement function are not part of any object boundary.

\subsection{Smoothness Constraints}

Problems related to missing data are common in the segmentation of medical structures. A solution to missing data is to exploit apriori knowledge by imposing smoothness constraints on the evolving surface. We use two types of smoothness 
constrains in this work, one on the speed of neighboring rays, the other on the local curvature of the front.

Thus, the speed function $S_{0}(x, y, z)$ of a ray is filtered by employing the speed information in a neighborhood. In addition, the speed term, $S_{1}(x, y, z)$ imposes the smoothness of the front. Currently, we employ the mean curvature given by $S_{1}(x, y, z)=\frac{\kappa_{1}+\kappa_{2}}{2}$ where $\kappa_{1}$ and $\kappa_{2}$ are the principal curvatures. However, other geometric smoothing techniques, such as the Mean-Gaussian curvature [11] can be used.

\subsection{Implementation}

For the initialization of rays we use an algorithm that provides an approximation to equidistant placement of points on a sphere by employing several subdivisions of an octahedron. The octahedron is initialized with the six points $(1,0,0)$, $(-1,0,0),(0,1,0),(0,-1,0),(0,0,1)$ and $(0,0,-1)$ and connections between neighboring points give the first primitive triangulization of the unit sphere. In the subdivision process each triangle is subdivided into four new ones by placing one new point on the middle of every edge, projecting it onto the unit sphere and connecting the neighboring points. Rays are then shot from the seed point along the direction given by this approximation of the unit sphere.

To estimate the surface curvature at the current position of a ray we use the following algorithm 202]: Compute the first matrix $\mathcal{S}$

$$
\mathcal{S}=\left(\frac{1}{2 N} \sum_{i=1}^{N} \mathbf{n}_{i} \mathbf{n}_{i}^{t}\right)+\frac{1}{2} \mathbf{p p}^{t}
$$

where $N$ is the number of neighbors, $\mathbf{n}_{i}$ denotes the vector to the current position of the $i$-th neighbor and $\mathbf{p}$ is the vector to the current position of the ray in question. The positive definite matrix $\mathcal{S}$ is called structure tensor or scatter matrix. A principle component analysis provides the eigenvalues $\sigma_{1} \geq \sigma_{2} \geq \sigma_{3}$ and eigenvectors $\mathbf{v}_{1}, \mathbf{v}_{2}, \mathbf{v}_{3}$ of $\mathcal{S}$. The eigenvector $\mathbf{v}_{1}$ corresponding to the largest eigenvalue $\sigma_{1}$ is in normal tangential direction. The other two eigenvectors point into the directions of the principal curvatures. Though the corresponding eigenvalues $\sigma_{2}$ and $\sigma_{3}$ already give an estimate of the degree of curvature in these directions they do not give information of their orientation, forcing us to include another step. Therefore, for every neighbor we also calculate the dot product of the neighbor vector and the principal curvature vector. This coefficient is then multiplied with the difference of $\frac{\pi}{2}$ and the angle between the normal tangential vector $\mathbf{v}_{1}$ and the vector connecting $\mathbf{p}$ and the neighbor. The sum over this weighted angle differences is positive for convex areas and negative for concave areas. Hence, the final formulas for principal curvatures are: $\kappa_{1}=\sum_{i=1}^{N} \frac{\left|\mathbf{v}_{2} \cdot \mathbf{n}_{\mathbf{i}}\right|}{\left|\mathbf{v}_{2}\right|\left|\mathbf{n}_{\mathbf{i}}\right|}\left(\arccos \left(\frac{\mathbf{v}_{1} \cdot\left(\mathbf{n}_{i}-\mathbf{p}\right)}{\left|\mathbf{v}_{1}\right|\left|\mathbf{n}_{i}-\mathbf{p}\right|}\right)-\frac{\pi}{2}\right)$ and $\kappa_{2}=\sum_{i=1}^{N} \frac{\left|\mathbf{v}_{3} \cdot \mathbf{n}_{\mathbf{i}}\right|}{\left|\mathbf{v}_{3}\right|\left|\mathbf{n}_{\mathbf{i}}\right|}\left(\arccos \left(\frac{\mathbf{v}_{1} \cdot\left(\mathbf{n}_{i}-\mathbf{p}\right)}{\left|\mathbf{v}_{1}\right|\left|\mathbf{n}_{i}-\mathbf{p}\right|}\right)-\frac{\pi}{2}\right)$.

\section{Results and Conclusions}

In this paper, we have presented a user-friendly $3 \mathrm{D}$ volume segmentation algorithm. Figures [1] and [2] show the segmentation of the inner boundary of an 
(a)

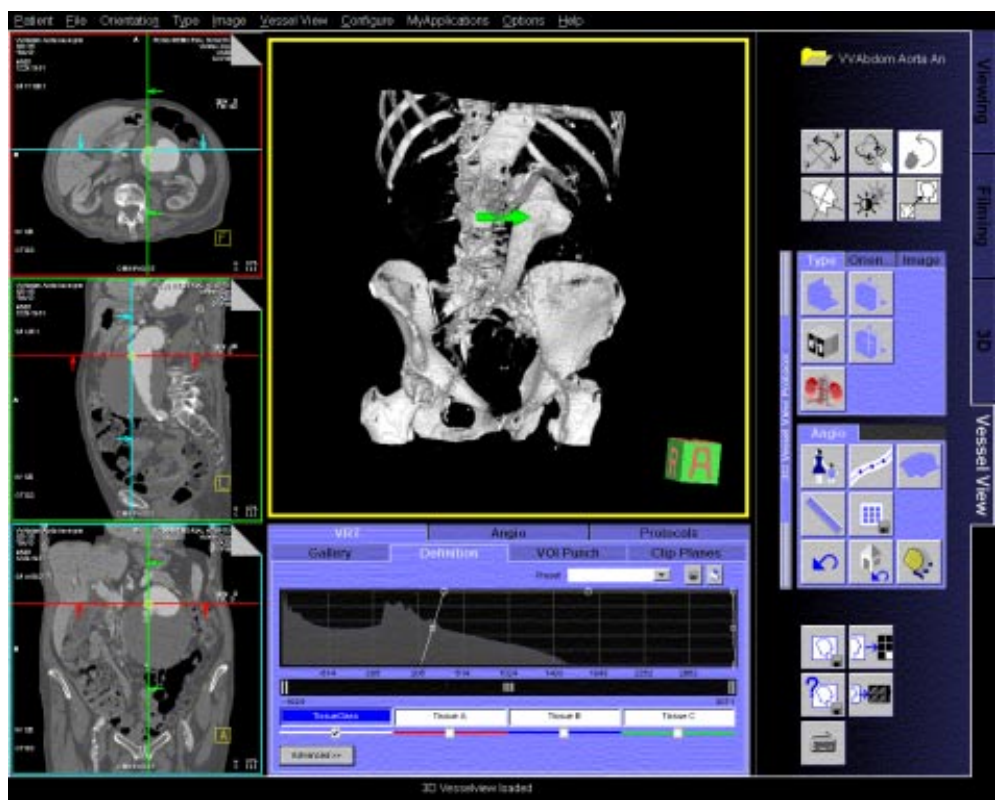

(b)

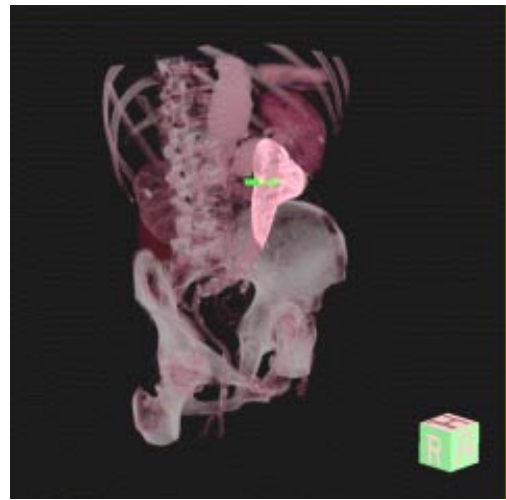

(c)

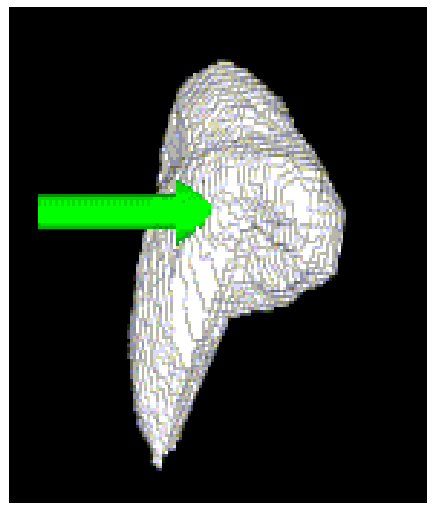

Fig. 2. This figure illustrates one-click segmentation algorithm. (a) illustrates the original image (CE-CTA) image in multi-planar reformats (MPRs) and volume rendering. This data set contains an aneurysm which is indicated by the (green) arrow. (b) The detected aneurysm (lumen boundary) is blended with the original data. (c) The segmented aneurysm (lumen boundary).

aneurysm on CE-CTA data. This proposed algorithm is very fast due to ray propagation. Second, the analysis based on mean shift makes our algorithm robust to outliers inherent in CT and MR images. Third, the use of shape priors such as smoothness constraints implies a reduced sensitivity of the algorithm to missing data, i.e., surfaces that are not well defined or missing. Fourth, our algorithm is user-friendly since one-click inside the 3D medical structure is often sufficient. 


\section{References}

1. D. Adalsteinsson and J. Sethian. A fast level set method for propagating interfaces. J. Comput. Phys, 118:269-277, 1995.

2. J. Berkmann and T. Caelli. On the relationship between surface covariance and differential geometry.

3. M. Black, G. Sapiro, D. Marimont, and D. Heeger. Robust anisotropic diffusion. 7:421-432, 1998 .

4. V. Caselles, F. Catte, T. Coll, and F. Dibos. A geometric model for active contours in image processing. TR No 9210, CEREMADE, 1992.

5. L. Cohen and I. Cohen. Finite element methods for active contour models and balloons for 2-d and 3-d images, 1993.

6. D. Comaniciu and P. Meer. Mean shift analysis and applications. In ICCV, pages 1197-1203, 1999.

7. D. Comaniciu and P. Meer. Mean shift: A robust approach toward feature space analysis. IEEE Trans. on PAMI, 24(5):To appear, 2002.

8. D. DeCarlo and D. N. Metaxas. Blended deformable models. IEEE Transactions on Pattern Analysis and Machine Intelligence, 18(4):443-448, 1996.

9. R. Malladi, J. A. Sethian, and B. C. Vemuri. Shape modelling with front propagation: A level set approach. IEEE Trans. on PAMI, 17, 1995.

10. T. McInerney and D. Terzopoulos. A dynamic finite element surface model for segmentation and tracking in multidimensional medical images with application to cardiac 4D image analysis. Comp. Med. Imaging and Graphics, 19:69-83, 1995.

11. P. Neskovic and B. B. Kimia. Three-dimensional shape representation from curvature-dependent deformations. TR-128, LEMS, Brown University, 1993.

12. S. Osher and J. A. Sethian. Fronts propagating with curvature dependent speed: Algorithms based on Hamilton-Jacobi formulations. J. of Comp. Phy, 79, 1988.

13. T. B. Sebastian, H. Tek, J. J. Crisco, S. W. Wolfe, and B. B. Kimia. Segmentation of carpal bones from $3 \mathrm{~d}$ CT images using skeletally coupled deformable models. In MICCAI, pages 1184-1194, 1998.

14. J. A. Sethian. Level Set Methods and Fast Marching Methods. Cambridge University Press, Second Ed., New York, 1999.

15. K. Siddiqi, A. Tannenbaum, and S. Zucker. Area and length minimizing flows for image segmentation. IEEE Trans. Image Processing, 7:433-444, 1998.

16. H. Tek, D. Comaniciu, and J. Williams. Vessel detection by mean shift based ray propagation. In Work. on Math. Models in Biomedical Image Analysis, 2001.

17. H. Tek and B. B. Kimia. Volumetric segmentation of medical images by threedimensional bubbles. CVIU, 64(2):246-258, February 1997.

18. D. Terzopoulos and D. Metaxas. models with local and global deformations : Deformable superquadrics. IEEE PAMI, vol. 13, no. 7, pp. 703-714, 1991., 1991.

19. C. Tomasi and R. Manduchi. Bilateral filtering for gray and color images. In ICCV, Bombay, India, pages 839-846, January 1998.

20. B. Vemuri, A. Mitiche, and J. Aggarwal. Curvature-based representation of objects from range data. Image and Vision Computing, 4:107-114, 1986.

21. R. Whitaker. Algorithms for implicit deformable models. In ICCV95, pages 822827, 1995.

22. R. Whitaker, D. Breen, K. Museth, and N. Soni. Segmentation of biological volume datasets using a level set framework. Volume Graphics, pages 249-263, 2001.

23. C. Xu and J. Prince. Snakes, shapes, and gradient vector flow. IEEE Trans. Image Proc, pages 359-369, 1998.

24. A. Yezzi and S. Kichenassamy and A. Kumar and P. Oliver and A. Tannenbaum" A Geometric Snake Model for Segmentation of Medical Imagery IEEE Transactions on Medical Imaging, 16(2), 1997. 